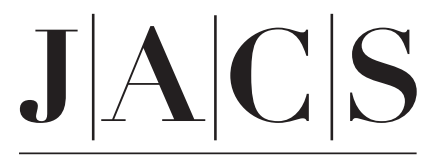

A R T I C L E S

Published on Web 04/09/2003

\title{
Measurement of Submicrosecond Intramolecular Contact Formation in Peptides at the Single-Molecule Level
}

\author{
Hannes Neuweiler, ${ }^{\dagger}$ Andreas Schulz, ${ }^{\dagger}$ Martin Böhmer,${ }^{\ddagger}$ Jörg Enderlein, ${ }^{*}, \neq$ and \\ Markus Sauer*,† \\ Contribution from the Physikalisch-Chemisches Institut, Universität Heidelberg, \\ Im Neuenheimer Feld 253, 69120 Heidelberg, Germany, and IBI-1, Forschungszentrum Jülich, \\ 52428 Jülich, Germany
}

Received January 5, 2003; E-mail: j.enderlein@fz-juelich.de; sauer@urz.uni-heidelberg.de

\begin{abstract}
We describe a single-molecule-sensitive method to determine the rate of contact formation and dissociation between tryptophan and an oxazine derivative (MR121) on the basis of measurements of the photon distance distribution. Two short peptides (15 and 20 amino acids) derived from the transactivation domain of the human oncoprotein p53 were investigated. With the fluorophore attached at the N-terminal end of the flexible peptides, fluorescence of the dye is efficiently quenched upon contact formation with a tryptophan residue. The mechanism responsible for the efficient fluorescence quenching observed in the complexes is assumed to be a photoinduced electron-transfer reaction occurring predominantly at van der Waals contact. Fluorescence fluctuations caused by intramolecular contact formation and dissociation were recorded using confocal fluorescence microscopy with two avalanche photodiodes and the time-correlated single-photon-counting technique, enabling a temporal resolution of $1.2 \mathrm{~ns}$. Peptides containing a tryptophan residue at positions 9 and 8 , respectively, show contact formation with rate constants of $1 / 120$ and $1 / 152$ $\mathrm{ns}^{-1}$, respectively. Whereas the rate constants of contact formation most likely directly report on biopolymer chain mobility, the dissociation rate constants of $1 / 267$ and $1 / 742 \mathrm{~ns}^{-1}$, respectively, are significantly smaller and reflect strong hydrophobic interactions between the dye and tryptophan. Fluorescence experiments on point-mutated peptides where tryptophan is exchanged by phenylalanine show no fluorescence quenching.
\end{abstract}

\section{Introduction}

Conformational flexibility is an important property of proteins and peptides to form stable folded structures. ${ }^{1}$ While folded proteins are routinely characterized with various powerful techniques, comparably little is known about unfolded proteins and their conformational fluctuations. A fundamental process in the dynamics of an unfolded peptide chain is the formation of amino acid contacts. The stability of these contact formations is determined by the structural properties of the corresponding amino acids, such as hydrophobicity and charge, as well as by molecular interactions with residues in other parts of the chain., ${ }^{2,3}$ To understand protein folding, it is essential to know the underlying mechanisms and time scales (extending from seconds to femtoseconds) of these elementary processes. ${ }^{4-6}$

To directly monitor nanosecond to microsecond processes, for example, the intramolecular contact formation in peptides

\footnotetext{
$\dagger$ Universität Heidelberg.
}

$\div$ IBI- 1

(1) Eaton, W. A.; Munoz, V.; Hagen, S. J.; Jas, G. S.; Lapidus, L. J.; Henry, E. R.; Hofrichter, J. Annu. Rev. Biophys. Biomol. Struct. 2000, 29, 327359.

(2) Chakrabartty, A.; Baldwin, R. L. Adv. Protein Chem. 1995, 46, 141-176.

(3) Munoz, V.; Serrano, L. Curr. Opin. Biotechnol. 1995, 6, 382-386.

(4) Frauenfelder, H.; McMahon, B. H. Annu. Phys. 2000, 9, 655-667.

(5) Agarwal, R.; Krueger, B. P.; Scholes, G. D.; Yang, M.; Yom, J.; Mets, L.; Fleming, G. R. J. Phys. Chem. B 2000, 104, 2908-2918.

(6) Woutersen, S.; Mu, Y.; Stock, G.; Hamm, P. Proc. Natl. Acad. Sci. U.S.A. 2001, 98, 11254-11258. or DNA hairpins, different strategies can be used. On one hand, "intelligent" fluorescent probes that can report information about their environment can be used, e.g., fluorescent dyes that are selectively quenched by DNA nucleotides. ${ }^{7,8}$ However, the fluorescence lifetimes of common fluorophores are typically in the range of several nanoseconds, i.e., too short to monitor nanosecond to microsecond processes. To circumvent this problem, long-living triplet-state probes have recently been introduced to measure fluctuation dynamics in polypeptides in the nanosecond to microsecond range by means of transient absorption spectroscopy. ${ }^{9-12}$ Measuring the lifetime of the tryptophan (Trp) triplet state, Lapidus et al. studied the loopclosure kinetics of peptides Cys-(Ala-Gly-Gln) $)_{n}$ Trp $(n=$ 1-6). ${ }^{11}$ With Trp at one end of the peptide and cysteine as efficient triplet quencher at the other, rate constants of end-toend contact formation were found to decrease from $\sim 1 / 40 \mathrm{~ns}^{-1}$ for $n=1$ to $\sim 1 / 140 \mathrm{~ns}^{-1}$ for $n=6$. On the other hand, pump-

(7) Seidel, C. A. M.; Schulz, A.; Sauer, M. J. Phys. Chem. 1996, 100, 55415533.

(8) Knemeyer, J. P.; Marmé, N.; Sauer, M. Anal. Chem. 2000, 72, 37173724.

(9) Hagen, J. S.; Hofrichter, J.; Szabo, A.; Eaton, W. A. Proc. Natl. Acad. Sci. U.S.A. 1996, 93, 11615-11617.

(10) McGimpsey, W. G.; Chen, L.; Carraway, R.; Samaniego W. N. J. Phys. Chem. A 1999, 103, 6082-6090.

(11) Lapidus, L. J.; Eaton, W. A.; Hofrichter, J. Proc. Natl. Acad. Sci. U.S.A. 2000, $97,7220-7225$.

(12) Hudgins, R. R.; Huang, F.; Gramlich, G.; Nau, W. M. J. Am. Chem. Soc. 2002, $124,556-564$.

5324 I J. AM. CHEM. SOC. 2003, 125, 5324-5330

10.1021/ja034040p CCC: $\$ 25.00$ @ 2003 American Chemical Society 
probe transient absorption experiments require high peptide concentrations, i.e., $10-100 \mu \mathrm{M}$, and the samples have to be deoxygenated to prevent quenching by oxygen and cross-linking of thiol groups, as well as to minimize photobleaching. In addition, UV excitation promotes photodamage especially under the required high-laser-intensity conditions. ${ }^{11}$ Hudgins et al. used 2,3-diazabicyclo[2.2.2] oct-2-ene (DBO) as a fluorophore with an extremely long fluorescence lifetime of several hundreds of nanoseconds which is efficiently quenched by $\operatorname{Trp}$ residues. ${ }^{13}$ Using polypeptides containing both a DBO residue and a tryptophan residue, the rate constants of contact formation were measured through the intramolecular fluorescence quenching of DBO by Trp with time-correlated single-photon counting (TCSPC). Unfortunately, the extinction of DBO is too low to reach the optimum sensitivity of fluorescence measurements (nanomolar to picomolar level), i.e., single-molecule sensitivity. ${ }^{13}$

Nowadays, single fluorescent dye molecules can be easily detected in solution and on surfaces with high signal-tobackground ratio. Single-molecule spectroscopy (SMS) is suited to study reaction pathways of individual members in a heterogeneous mixture and follow fluctuations that are impossible to synchronize at the ensemble level. ${ }^{14-18}$ The ability to record and analyze trajectories of individuals is also crucial in many biological systems. Examples range from enzyme reactions with proposed multiple conformational substates ${ }^{19,20}$ and protein folding with multiple unfolded states and pathways ${ }^{21}$ to interactions between cell surface receptors or components of signal transduction pathways. ${ }^{22}$ Furthermore, interactions between proteins, DNA, and RNA or conformational dynamics of single DNA/RNA or protein molecules can be studied at the level of single molecules. ${ }^{23,24}$ This was made possible not only by the development of optical single-molecule techniques but likewise through the application of single-pair fluorescence resonance energy transfer (spFRET) techniques. ${ }^{17,25}$ Förster-type resonance energy transfer occurs in the distance range of $2-8 \mathrm{~nm}$ for allowed singlet-singlet transitions and sufficient spectral overlap of donor emission and acceptor absorption. Due to its strong distance dependence, spFRET is ideally suited to obtain information about the structural dynamics of biologically important molecules on the microsecond to millisecond time scale. ${ }^{23,24,26-29}$

(13) Hudgins, R. R.; Huang, F.; Gramlich, G.; Nau, W. M. J. Am. Chem. Soc 2002, 124, 556-564

(14) Xie, X. S.; Trautman, J. K. Annu. Rev. Phys. Chem. 1998, 49, 441-480.

(15) Funatsu, T.; Harada, Y.; Tokunaga, M.; Saito, K.; Yanagida, T. Nature (London) 1995, 374, 555-559.

(16) Ishijima, A.; Kojima, H.; Funatsu, T.; Tokunaga, M.; Higuchi, H.; Tanaka, H.; Yanagida, T. Cell 1998, 92, 161-171.

(17) Weiss, S. Science 1999, 283, 1676-1683.

(18) Moerner, W. E.; Orrit, M. Science 1999, 283, 1670-1676.

(19) Frauenfelder, H.; Silgar, S. G.; Wolynes, P. G. Science 1991, 253, 15981603.

(20) Radar, S. D.; Agard, D. A. Protein Sci. 1997, 6, 1375-1386.

(21) Leeson, D. T.; Gai, F.; Rodriguez, H. M.; Gregoret, L. M.; Dyer, R. B. Proc. Natl. Sci. U.S.A. 2000, 97, 2527-2532.

(22) Deller, M. C.; Jones, E. Y. Curr. Opin. Struct. Biol. 2002, 10, 213-219.

(23) Bonnet, G.; Krichevsky, O.; Libchaber, A. Proc. Natl. Acad. Sci. U.S.A 1998, 95, 8602-8606

(24) Wallace, M. I.; Ying, L. M.; Balasubramanian, S.; Klenerman, D. Proc. Natl. Acad. Sci. U.S.A. 2001, 98, 5584-5589.

(25) Ha, T.; Enderle, Th.; Ogletree, D. F.; Chemla, D. S.; Selvin, P. R.; Weiss, S. Proc. Natl. Acad. Sci. U.S.A. 1996, 93, 6264-6268.

(26) Ha, T.; Ting, A. Y.; Liang, J.; Caldwell, W. B.; Deniz, A. A.; Chemla, D. S.; Schultz, P. G.; Weiss, S. Proc. Natl. Acad. Sci. U.S.A. 1999, 96, 893898

(27) Jia, Y.; Malaga, D. S.; Lau, W. L.; Lui, H. S. M.; DeGrado, W. F.; Hochstrasser, R. M. Chem. Phys. 1999, 247, 69-83.
The kinetics of DNA hairpin-loop fluctuations are one of the fastest conformational fluctuations observed so far by singlemolecule spectroscopy. Using a combination of fluorescence resonance energy transfer and fluorescence correlation spectroscopy, the chemical rates for opening and closing of the DNA hairpins were determined to a few tens of microseconds dependent on the size and sequence of the loop and stem. ${ }^{23,24}$ However, this method has two important limitations. First, spFRET experiments require site-specific labeling of the polypeptide with two extrinsic probes, a donor and an acceptor dye. A method that takes advantage of properties of naturally occurring amino acids would be more useful. Second, the temporal resolution is restricted by the dead time of the avalanche photodiodes (APDs) commonly used as detectors (dead time values between $100 \mathrm{~ns}$ and $1 \mu$ s); i.e., the dead time of APDs precludes monitoring of fast intensity fluctuations in the time range $<1 \mu \mathrm{s}$.

We have developed a new method to measure fluorescence fluctuations in peptides at the single-molecule level that utilizes efficient fluorescence quenching of a fluorescent dye by $\operatorname{Trp}$ residues ${ }^{30}$ in combination with a new technique to measure the second-order fluorescence intensity correlation with nanosecond time resolution. The basic idea of the experiment is that the fluorescence of the excited fluorophore, an oxazine derivative (MR121), is efficiently quenched by Trp only upon contact formation. The fluorescence quenching of the dye by Trp is unique since it requires a close, well-defined molecular interaction. Except for tryptophan itself, all other amino acids quench with rates that are substantially smaller. ${ }^{31}$ To demonstrate the utility of this method, we investigated the rate of contact formation between an oxazine derivative attached at the $\mathrm{N}$-terminal end and a Trp residue within two peptides of 15 and 20 amino acid lengths. The peptides are linear fragments of the amino-terminal transactivation domain of human p53, which is the target for several protein partners such as the TATA box-binding protein (TBP), MDM2, or antibodies from the humoral immune response of cancer patients. ${ }^{32-35}$ The crystal structure of a 109-residue amino-terminal domain of MDM2 bound to p53 peptides revealed that MDM2 has a deep hydrophobic cleft on which the p53 peptide binds as amphipathic $\alpha$ helix. ${ }^{34}$ In the absence of MDM2, the $\alpha$ helices are readily digested by proteases, indicating that the peptides are only loosely folded, or folded in only a fraction of the molecules. ${ }^{34}$ Our data support the idea of an unfolded or only loosely folded peptide.

The general principle of the new fluorescence-based method for measuring the kinetics of intramolecular contact formation in biopolymers is shown in Figure 1. A fluorescent dye (MR121)

(28) Ha, T.; Zhuang, X.; Kim, H. D.; Orr, J. W.; Williamson, J. R.; Chu, S. Proc. Natl. Acad. Sci. U.S.A. 1999, 96, 9077-9082.

(29) Talaga, D. S.; Lau, W. L.; Roder, H.; Tang, J.; Jia, Y.; DeGrado, W. F.; Hochstrasser, R. M. Proc. Natl. Acad Sci. U.S.A. 2000, 97, 13021-13026.

(30) Kavarnos., G. J. Fundamentals of Photoinduced Electron Transfer; VCH Publishers: New York, 1993.

(31) Watt, R. M.; Voss, E. W. Immunochemistry 1977, 4, 533-541.

(32) Lubin, R.; Schlichtholz, B.; Bengoufa, D.; Zalcman, G.; Tre'daniel, T.; Hirsch, A.; Caron de Fromentel, C.; Preudhomme, C.; Fenaux, P.; Fournier, G.; Mangnin, P.; Laurent-Puig, P.; Pelletier, G.; Schlumberger, M.; Desgrandchamps, F.; Le Duc, A.; Peyrat, J. P.; Janin, N.; Bressac, B.; Soussi, T. Cancer Res. 1993, 53, 5872-5876.

(33) Neuweiler, H.; Schulz, A.; Vaiana, A. C.; Smith, J. C.; Kaul, S.; Wolfrum, J.; Sauer, M. Angew. Chem., Int. Ed. 2002, 41, 4769-4773.

(34) Kussie, P. H.; Gorina, S.; Marechal, V.; Elenbaas, B.; Moreau, J.; Levine, A. J.; Pavletich, N. P. Science 1996, 274, 948-953.

(35) Soussi, T. Immunol. Today 1996, 17 (8), 354-356. 


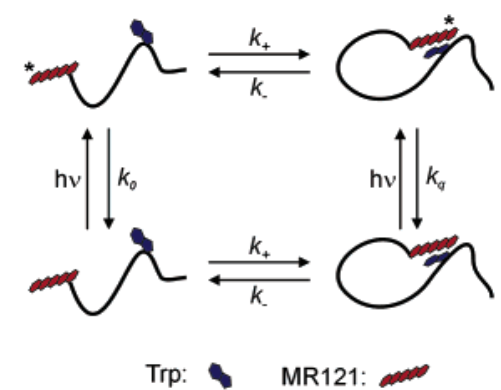

Figure 1. General principle behind using fluorescence quenching of dyes by $\operatorname{Trp}$ to measure peptide dynamics. The dye/Trp association and subsequent efficient fluorescence quenching are used to reveal contact formation rate constants. It should be noted that the diffusion rate constants in the excited state $(*)$ are assumed to resemble those in the ground state for the association rate $k_{+}$and for the dissociation rate $k_{-}$.

and the quencher Trp are incorporated into a peptide. The dynamics of the peptide backbone will lead to conformations where the dye and the Trp residue come in contact (top-right, and bottom-right structures). After excitation of the dye molecule, it either emits a fluorescence photon (dependent on the fluorescence quantum yield) or is quenched due to an interaction with the Trp residue. These two possibilities are reflected in the rate of the excited-state decay (fluorescence lifetime), $k_{0}$, and the intramolecular quenching rate constant, $k_{\mathrm{q}}$. If contact formation between the dye MR121 and the Trp residue is followed by immediate fluorescence quenching, the top-right structure in Figure 1 can be assumed to be practically nonfluorescent, comparable to an off state due to intersystem crossing into long-lived triplet states. Hence, second-order fluorescence intensity correlation can be used to reveal the rate for contact formation (association rate), $k_{+}$, as well as the dissociation rate, $k_{-}$.

We used optical excitation of the oxazine dye MR121 in the red spectral range to measure the Trp-induced fluorescence quenching upon contact formation by calculating the secondorder fluorescence intensity correlation from $10^{-10} \mathrm{M}$ peptide solutions. To achieve the desired time resolution in the nanosecond range, the signal was shared by two avalanche photodiodes using a 50:50 beam splitter and recorded by the TCSPC technique. Using the classical antibunching setup, ${ }^{36-38}$ the temporal interval distribution between fluorescence photons detected on the two APDs, i.e., the interphoton times, were registered with a time resolution of $1.2 \mathrm{~ns}$. Association (contact formation) and dissociation rate constants in the range of a few hundred nanoseconds were measured for two tryptophancontaining peptides. The exchange of the tryptophan by a phenylalanine residue results in the disappearance of the short correlation term in the $100 \mathrm{~ns}$ time range.

\section{Materials and Methods}

Synthesis of Fluorescently Labeled Peptides. N-terminal labeling of the peptides with the fluorescent dye MR121 was performed by classical $N$-hydroxysuccinimidyl ester (NHS ester) chemistry using standard solvents purchased from Merck (Darmstadt, Germany). The oxazine dye MR121 $(1 \mu \mathrm{mol} / \mathrm{mL})$ and the synthetic peptides $(1 \mu \mathrm{mol} /$ $\mathrm{mL}$ ) were dissolved in dimethylformamide (DMF). A $10 \mu \mathrm{L}(10 \mathrm{nmol})$

(36) Hanbury-Brown, R.; Twiss, R. Q. Nature (London) 1956, 177, 27-29.

(37) Basché, Th.; Moerner, W. E.; Oritt, M.; Talon, H. Phys. Rev. Lett. 1992 69, 1516-1519.

(38) Fleury, L.; Segura, J.-M.; Zumofen, G.; Hecht, B.; Wild, U. P. Phys. Rev. Lett. 2000, 84, 1148-1151. sample of the dye solution was added to $100 \mu \mathrm{L}$ (100 nmol, 10-fold excess) of the peptide and $2 \mu \mathrm{L}$ of diisopropylethylamine (DIPEA). The solution was incubated for $3 \mathrm{~h}$ at room temperature in the dark. The amino-terminal conjugate was purified by reversed-phase (HypersilODS column) HPLC (Agilent Technologies, Waldbronn, Germany) using a linear gradient of $0-75 \%$ acetonitrile in $0.1 \mathrm{M}$ aqueous triethylammonium acetate. Reaction yields of the amino-terminal products of about $55 \%$ were achieved. The purity of the probes was checked by capillary gel electrophoresis.

Fluorescence Ensemble Measurements. All measurements were performed at room temperature $\left(25^{\circ} \mathrm{C}\right)$ in an aqueous solution of phosphate-buffered saline (PBS; $\mathrm{pH}$ 7.4). Relative fluorescence quantum yields, $\Phi_{\text {f,rel }}$, of the labeled peptides were measured with respect to the fluorescence intensity of the peptide conjugates containing phenylalanine instead of tryptophan. Ensemble fluorescence lifetimes, $\tau$, were measured with a standard spectrometer from IBH (model $5000 \mathrm{MC}$; Glasgow, U.K.) for TCSPC using a pulsed diode laser $(635 \mathrm{~nm})$ as the excitation source (4096 channels, 12.5 ps, 5.000 photons in the maximum channel). To exclude polarization effects, fluorescence was observed under the magic angle $\left(54.7^{\circ}\right)$. The decay parameters were determined by least-squares deconvolution, and their quality was judged by the reduced $\chi^{2}$ values and the randomness of the weighted residuals. In case a monoexponential model was not adequate to describe the measured decay, a multiexponential model, $I(t)=I(0) \sum a_{i} \tau_{i}$, was used to fit the decay. Here $a_{i}$ are the preexponential factors that describe the ratio of the excited species, and $\tau_{i}$ denote their lifetimes.

Single-Molecule Experiments. To diminish glass surface adsorptions, all single-molecule measurements were performed in $100 \mathrm{mM}$ sodium phosphate buffer ( $\mathrm{pH} 7.4$ ) containing $0.3 \mathrm{mg} / \mathrm{mL}$ hen eggwhite albumin. Details of the experimental setup are described in ref 41 . To report briefly, a krypton-argon ion laser (Stabilite 2018, SpectraPhysics) at $647.1 \mathrm{~nm}$ was used as the excitation source. The laser light passes an excitation laser-line filter (647NB4, Omega Optics), and is directed into a high-aperture oil-immersion objective $(100 \times$, NA 1.4 , CFI; Nikon) by a dichroic mirror (650DRLP, Omega Optics). Sample fluorescence is collected by the same objective and transmitted through the dichroic mirror. A tube lens focuses the light onto a $100 \mu \mathrm{m}$ pinhole for confocal imaging. After the pinhole, the light is split by a polarizing beam splitter (TSWP 670, Linos Photonics), and two chromatic doublet lenses refocus the light onto the active area of two single-photon APDs (AQR-14, Perkin-Elmer) with quantum yields of $\sim 60 \%$ at $670 \mathrm{~nm}$. Two emission band-pass filters (690DF40, Omega Optics) discriminate fluorescence and scattered light. The TTL pulses of the APDs were recorded by time-correlated single-photon-counting electronics (TimeHarp200, Picoquant). The card measures the time distance between two photon detection events from the two APDs. The interphoton times are histogrammed in 4096 channels. The card parameters were set to measure interphoton times over a time range of $5 \mu \mathrm{s}$ with a temporal resolution of $1.2 \mathrm{~ns}$. The solutions for single-molecule experiments were prepared by diluting a stock solution $\left(10^{-6} \mathrm{M}\right)$ with the appropriate amount of buffer down to the required concentration of $10^{-10} \mathrm{M}$.

\section{Results and Discussion}

Fluorescence Quenching of MR121 by Trp Residues. To interpret intramolecular quenching experiments in terms of contact formation, bimolecular quenching experiments have to be performed. Among the 20 natural amino acids, only Trp quenches the fluorescence of the red-absorbing oxazine dye MR121 significantly. The quenching experiments yielded identical results, within error, in $\mathrm{PBS}, \mathrm{pH} 7.4$, and unbuffered $\mathrm{H}_{2} \mathrm{O}$.

(39) Böhmer, M.; Pampaloni, F.; Wahl, M.; Rahn, H. J.; Erdmann, R.; Enderlein, J. Rev. Sci. Instrum. 2001, 72, 4145-4152.

(40) Lee, S.; Winnik, M. A. Macromolecules 1997, 30, 2633-2641.

(41) Daugherty, D. L.; Gellman, S. H. J Am. Chem. Soc. 1999, 121, 43254333 . 
Table 1. Ensemble Spectroscopic Characteristics of the Oxazine Derivative MR121 and Labeled Peptide Conjugates MR121-SQETFSDLWKLLPEN (MR121-W9), MR121-SQETFSDLFKLLPEN (MR121-W9·F9), MR121-SPDDIEQWFTEDPGPDEAPR (MR121-W8), and MR121-SPDDIEQFFTEDPGPDEAPR (MR121-W8·F8) ${ }^{a}$

\begin{tabular}{|c|c|c|c|c|c|c|c|c|}
\hline & $\begin{array}{l}\lambda_{\text {abs }} \\
(\mathrm{nm})\end{array}$ & $\begin{array}{c}\lambda_{\mathrm{em}} \\
(\mathrm{nm})\end{array}$ & $\Phi_{\mathrm{t}, \mathrm{rel}}$ & $\begin{array}{c}\tau_{1} \\
\text { (ns) }\end{array}$ & $a_{1}$ & $\begin{array}{c}\tau_{2} \\
\text { (ns) }\end{array}$ & $a_{2}$ & $\chi^{2}$ \\
\hline MR121 & 661 & 673 & 0.82 & 1.85 & 1.00 & & & 1.066 \\
\hline MR121/50 mM Trp & 667 & 683 & 0.05 & 1.42 & 1.00 & & & 1.084 \\
\hline MR121-W9 & 666 & 683 & 0.31 & 0.56 & 0.06 & 2.11 & 0.94 & 1.107 \\
\hline MR121-W9·F9 & 664 & 676 & 1.00 & 0.61 & 0.11 & 2.27 & 0.89 & 1.105 \\
\hline MR121-W8 & 667 & 683 & 0.17 & 0.51 & 0.09 & 2.00 & 0.91 & 1.138 \\
\hline MR121-W8·F8 & 664 & 677 & 1.00 & 0.61 & 0.12 & 2.26 & 0.88 & 1.071 \\
\hline
\end{tabular}

${ }^{a}$ Measurements were performed in PBS at room temperature. $\lambda_{\mathrm{abs}}$ and $\lambda_{\mathrm{em}}$ are the absorption and emission maxima of the measured probes, $\Phi_{\mathrm{f}, \mathrm{rel}}$ denotes the relative fluorescence quantum yield, and $\tau_{i}$ are the fluorescence lifetimes with corresponding amplitudes, $a_{i}$. Abbreviations for the amino acid residues are A, alanine; D, aspartic acid; E, glutamic acid; F, phenylalanine; G, glycine; I, isoleucine; K, lysine; L, leucine; N, asparagine; P, proline; Q, glutamine; $\mathrm{R}$, arginine; $\mathrm{S}$, serine; $\mathrm{T}$, threonine; and $\mathrm{W}$, tryptophan.

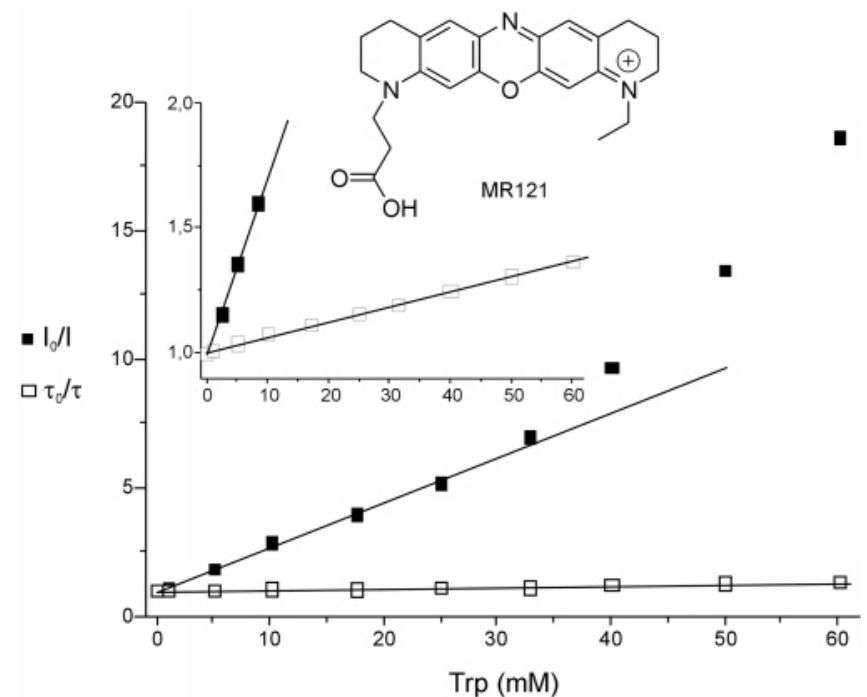

Figure 2. Static and dynamic bimolecular Stern-Volmer plot of MR121 and Trp in PBS, pH 7.4 (excitation wavelength $635 \mathrm{~nm}$, detection wavelength $680 \mathrm{~nm}$ ). The inset shows an expanded view.

We measured the steady-state absorption and fluorescence intensity as well as the fluorescence lifetime of free MR121 as a function of Trp concentration (Figure 2), yielding a relatively small bimolecular dynamic quenching rate, $k_{\mathrm{q}, \mathrm{d}}$, of $3.0 \times 10^{9}$ $\mathrm{M}^{-1} \mathrm{~s}^{-1}$ though the observed decrease in fluorescence intensity is much more pronounced. In addition, the absorption and emission maxima of MR121 show significant bathochromic shifts upon addition of Trp (Table 1). The Stern-Volmer plots obtained from steady-state experiments display a upward curvature, indicating the existence of at least two populations of fluorophores. Comparison of the bimolecular static quenching rate, $k_{\mathrm{q}, \mathrm{st}}$, of $11.9 \times 10^{10} \mathrm{M}^{-1} \mathrm{~s}^{-1}$ calculated from the linear region of the plot with the estimated diffusion-controlled bimolecular rate constant $\left(\sim 6.5 \times 10^{9} \mathrm{M}^{-1} \mathrm{~s}^{-1}\right)$ demonstrates the formation of nonfluorescent or only weakly fluorescent ground-state complexes between MR121 and Trp. The fact that the fluorescence lifetime of MR121 remains monoexponential in the presence of $\operatorname{Trp}$ indicates that these complexes are essentially nonfluorescent; i.e., they exhibit a fluorescence lifetime shorter than the time resolution of the instrument $(\sim 40$ ps) used to measure the fluorescence decays. Considering the bimolecular dynamic quenching rate, we extracted an association constant, $K_{\mathrm{S}}$, for complex formation of $\sim 220 \mathrm{M}^{-1}$ from the static Stern-Volmer plot.

The intramolecular quenching efficiency was measured for both peptides with MR121 at the N-terminus and either Trp

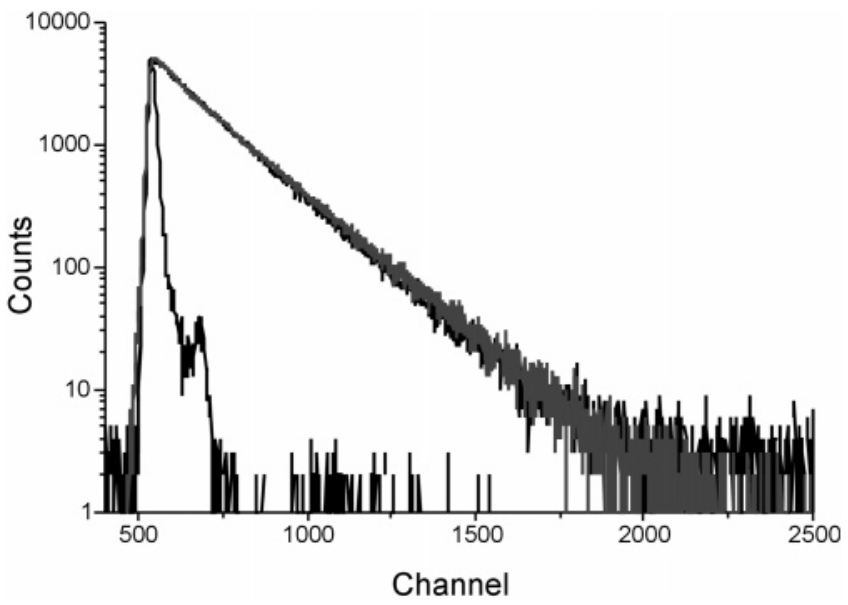

Figure 3. Fluorescence decay of Phe-containing peptide MR121-W9·F9 (gray) and the Trp-containing peptide MR121-W9 (black) in water excited at $635 \mathrm{~nm}$ together with the instrument response function (IRF). Fluorescence was monitored at $680 \mathrm{~nm}(12 \mathrm{ps} /$ channel)

(W) or Phe $(\mathrm{F})$ at position 8 or 9 , respectively, i.e., peptides MR121-W8, MR121-W8·F8, MR121-W9, and MR121W9.F9 (Table 1). As in the intermolecular quenching experiments, the absorption and emission maxima show slight red shifts especially in the Trp-containing peptides. Figure 3 shows the fluorescence decays of peptide MR121-W9 and MR121W9·F9. The pure dye MR121 exhibits a monoexponential fluorescence lifetime of $1.85 \mathrm{~ns}$ under the experimental conditions. The two peptides show similar fluorescence decays with approximately $10 \%$ of a shorter fluorescence lifetime of $\sim 500-$ $600 \mathrm{ps}$, and a main component with a lifetime slightly longer than the lifetime of the free dye of $\sim 2 \mathrm{~ns}$. In contrast, the fluorescence quantum yields reflect the expected selective fluorescence quenching of MR121 by Trp residues (Table 1). There is a dramatic decrease in fluorescence quantum yield for peptides containing Trp (peptides MR121-W8 and MR121W9). On the other hand, the fluorescence quantum yield of MR121 is slightly increased in peptides MR121-W8.F8 and MR121-W9·F9. This implies the formation of complexes between Trp and MR121 that exhibit very efficient fluorescence quenching. Such ultrafast processes fall beyond the detection limit of our experimental setup. Hence, we can assume those complexes as essentially nonfluorescent. The lifetime component of 500-600 ps can be well ascribed to slight quenching of the other amino acids.

Quenching Mechanism. Inter- and intramolecular quenching experiments indicate that the underlying quenching mechanism 
is only efficient at short distances, i.e., within the complexes. This is corroborated by the observation that the static quenching rate, $k_{\mathrm{q}, \mathrm{st}}$, strongly decreases upon addition of denaturing agents such as urea or guanidium chloride. Therefore, it can be concluded that hydrophobic interactions between MR121 and Trp play an important role in the formation of these nonfluorescent ground-state complexes. ${ }^{40,41}$ Fluorescence experiments in $\mathrm{D}_{2} \mathrm{O}$ did not give any evidence for the involvement of hydrogen bonds in fluorescence quenching. As collisional quenching is inefficient $\left(k_{\mathrm{q}, \mathrm{d}},=3.0 \times 10^{9} \mathrm{M}^{-1} \mathrm{~s}^{-1}\right)$, a stacked arrangement of the indole ring and the oxazine ring system in the complex appears to be responsible for ultrafast fluorescence quenching. ${ }^{42,43}$ Most likely, the mechanism involves a photoinduced charge-transfer interaction between the Trp and MR121. Photoinduced electron transfer from electron-rich donors such as the DNA base guanosine to excited rhodamine and oxazine dyes is a well-known process..$^{7,8,44,45}$ Electron-transfer reactions are controlled by the relation between the free energy of the reaction, the reorganization energy, and the distance between the donor and acceptor. ${ }^{46,47}$ Furthermore, electron-transfer reactions do not require intimate molecular contact and may well occur over larger distances, e.g., through space or through bond by a superexchange mechanism. ${ }^{48}$ Hence, the measured rate constants may not directly reflect intrachain contact formation. However, the bimolecular quenching constants point out that efficient quenching via electron transfer occurs only at van der Waals contact $(\sim 0.4 \mathrm{~nm})$.

Trp is known as a strong intrinsic electron donor in peptides with an oxidation potential of $\sim 0.80-1.00 \mathrm{~V}$ vs SCE at neutral $\mathrm{pH},{ }^{49,50}$ i.e., even lower than guanosine $\left(E_{\mathrm{ox}}=1.25 \mathrm{vs} \mathrm{SCE}\right){ }^{7}$ Unfortunately, the reduction potential, $E_{\text {red, of MR121 is not }}$ available. However, $E_{\text {red }}$ can be estimated roughly from measured values for several, structurally related red-absorbing rhodamine derivatives to approximately $-0.5 \mathrm{~V}$ vs SCE. ${ }^{51}$ With a transition energy, $E^{*}$, of $\sim 1.9 \mathrm{eV}$ for MR121 the free energy change for photoinduced electron transfer, $\Delta G^{\circ}$, from the ground-state Trp to the excited oxazine chromophore at infinite separation can be estimated to be approximately $-0.5 \mathrm{eV}$. On the other hand, it has been proposed that electron-transferinduced fluorescence quenching of end-labeled probes by a terminal Trp in peptides becomes important only when the excited-state reduction potential $\left(E_{\text {red }}+E^{*}\right)$ exceeds a value of $1.5 \mathrm{~V} .{ }^{52}$ Hence, the electron-transfer reaction between Trp and MR121 is, if any, only slightly exergonic in peptides, consistent with the small bimolecular dynamic quenching rate obtained

(42) Zhong, D.; Zewail, A. H. Proc. Natl. Acad. Sci. U.S.A. 2001, 98, 1186711872 .

(43) Malaga, N.; Chosrowjan, H.; Shibata, Y.; Tanaka, F.; Nishina, Y.; Shiga, K. J. Phys. Chem. B 2000, 104, 10667-10677.

(44) Sauer, M.; Drexhage, K. H.; Lieberwirth, U.; Müller, R.; Nord, S.; Zander, C. Chem. Phys. Lett. 1998, 284, 153-163.

(45) Wennmalm, S.; Edman, L.; Rigler, R. Proc. Natl. Acad. Sci. U.S.A. 1997 94, 10641-10646.

(46) Rehm, D.; Weller, A. Isr. J. Chem. 1970, 8, 259-271.

(47) Marcus, R. A.; Sutin, N. Biochim. Biophys. Acta 1985, 811, 265-320.

(48) Jones I., G.; Lu, L. N.; Fu, H.; Farahat, C. W.; Oh, C.; Greenfield, S. R.; Gosztola, D. J.; Wasielewski, M. R. J. Phys. Chem. B 1999, 103, 572581 .

(49) Wagenknecht, H. A.; Stemp, E. D. A.; Barton, J. K. J Am. Chem. Soc. 2000, 122, 1-7.

(50) DeFelippis, M. R.; Murthy, C. P.; Broitman, F.; Weinraub, D.; Faraggi, M.; Klapper, M. H. J. Phys. Chem. 1991, 95, 3416-3419.

(51) Sauer, M.; Han, K. T.; Müller, R.; Nord, S.; Schulz, A.; Seeger, S.; Wolfrum, J.; Arden-Jacob, J.; Deltau, G.; Marx, N. J.; Zander, C.; Drexhage, K. H. J. Fluoresc. 1995, 5, 247-261.

(52) Jones, G.; Lu, L. N.; Vullev, V.; Gosztola, D. J.; Greenfield, S. R.; Wasielewski, M. R. Bioorg. Med. Chem. Lett. 1995, 5, 2385-2390.

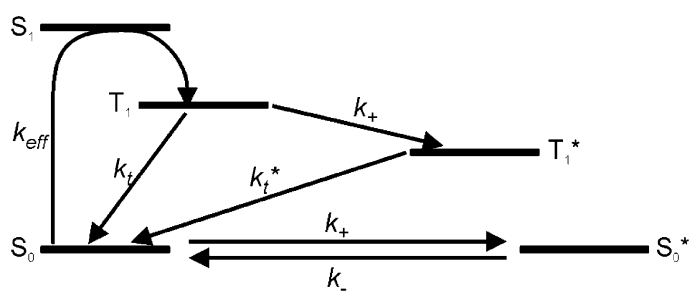

Figure 4. Simplified energetic scheme for the photophysics of the dye MR121 when attached covalently to a polypeptide containing a Trp residue as an efficient fluorescence quencher.

from intermolecular measurements. This demonstrates that the formation of ground-state complexes between Trp and MR121 is the primary prerequisite for efficient electron transfer. These unique quenching properties of the donor/acceptor pair Trp/ MR121, i.e., the contact-induced efficient quenching process, can be used to monitor the rate of intramolecular contact formation in peptides.

Single-Molecule Fluorescence Fluctuation Analysis. Generally, the photophysics of fluorescent dyes such as MR121 is described by a three-state model, comprising the ground state, $S_{0}$, the first excited singlet state, $S_{1}$, and the first excited triplet state, $T_{1}$. If the dye is in $S_{1}$ or $T_{1}$ during association with Trp, a fast electron transfer occurs from the Trp residue to $S_{0}$ of the dye, followed by either a fast reverse electron transfer of the $\mathrm{S}_{1}$ electron to Trp or a slow relaxation of the $\mathrm{T}_{1}$ state. Furthermore, we assume that the transition from $S_{1}$ to $S_{0}$, as well as the electron-transfer processes, occurs on a time scale faster than that of the nanosecond temporal resolution of the setup used. Then the observable photodynamics of fluorescence can be described by the reduced scheme shown in Figure 4. Upon excitation of the dye MR121, the triplet state $\mathrm{T}_{1}$ is populated with an effective rate $k_{\text {eff }}=k_{\text {exc }} k_{\text {ics }} /\left(k_{10}+k_{\text {ics }}\right)$, where $k_{\text {exc }}$ is the local excitation rate (excitation intensity times absorption cross section), $k_{\text {isc }}$ is the intersystem crossing rate, and $k_{10}$ is the transition rate from $S_{1}$ to $S_{0}$. The rate constant for contact formation, i.e., the association rate, is given by $k_{+}$. If contact formation takes place in the excited state $S_{1}$ or ground state $S_{0}$, it automatically ends in a nonfluorescent ground state, $\mathrm{S}_{0}$ *; if contact formation occurs in the triplet state $\mathrm{T}_{1}$, the dye is transferred into a modified triplet state (radical anion state), $\mathrm{T}_{1}{ }^{*}$, from where it relaxes with a modified rate, $k_{\mathrm{t}}{ }^{*}$, to $\mathrm{S}_{0}$. Here, $k_{\mathrm{t}}^{*}$ is understood as an effective rate constant comprising both the dissociation of the dye from the Trp residue and relaxation to $\mathrm{S}_{0}$ as well as relaxation to $\mathrm{S}_{0} *$ and subsequent dissociation from the Trp residue. As long as the dye stays in $\mathrm{S}_{0}{ }^{*}$, its fluorescence is assumed to be completely quenched via an efficient electron-transfer reaction.

Denoting by $s_{0}, t_{1}, t_{1} *$, and $s_{0} *$ the probabilities to find the dye in the corresponding states, the kinetic equations are given by

$$
\begin{gathered}
\dot{s}_{0}=-\left(k_{\mathrm{eff}}+k_{+}\right) s_{0}+k_{\mathrm{t}} t_{1}+k_{1} * t_{1} *+k_{-} s_{0}{ }^{+} \\
\dot{t}_{1}=k_{\mathrm{eff}} s_{0}-\left(k_{\mathrm{t}}+k_{+}\right) t_{1} \\
\dot{t}_{0}=k_{+} t_{1}-k_{1} * t_{1} * \\
\dot{s}_{0}^{*}=k_{+} s_{0}-k_{-} s_{0} *
\end{gathered}
$$

During a measurement, the temporal interval distribution between subsequently detected photons is measured, resulting 
in a photon distance distribution (PDD). Measuring temporal interval distances between subsequent photon detection events is similar to measuring the fluorescence autocorrelation in fluorescence correlation spectroscopy, with the additional restriction that between the two photon detection events no additional photons are detected. The detection of the first photon conveys the information that the dye molecule has just returned to the ground state $S_{0}$ at the moment of detection. The probability to detect the next photon after time $t$ is directly proportional to the probability $s_{0}(t)$ to find the dye still in $\mathrm{S}_{0}$ (in the simplified scheme of Figure 4, the fluorescence emission rate is directly proportional to $s_{0}(t)$, provided that the excitation intensity is far below optical saturation). With the initial conditions $s_{0}(0)=1, t_{1}(0)=0, t_{1} *(0)=0$, and $s_{0} *(0)=0$, the solution for $s_{1}$ is found from eq 1 in a standard way as

$$
s_{1}(t)=c_{0}+\sum_{j=1}^{3} c_{j} \exp \left(-\lambda_{j} t\right)
$$

where the $\lambda_{j}$ are the nonzero roots of the characteristic equation of eq 1 (one of the characteristic roots is always zero, reflecting the fact that the sum of all state population probabilities always remains one, and is associated with the constant $c_{0}$ in the last equation). In the case where there is no MR121/Trp association and thus no fluorescence quenching, only one physically meaningful and nonzero root remains, given by $\lambda_{1}=k_{\mathrm{t}}+k_{\mathrm{eff}}$. The photon distance distribution, $g(t)$, i.e., the probability to detect a photon at time $t$ after having detected a photon at zero time with no detection event between is then proportional to

$$
g(t) \propto \epsilon s_{1}(t) \exp \left[-\epsilon \int_{0}^{t} \mathrm{~d} t^{*} s_{1}\left(t^{*}\right)\right] \approx \epsilon S_{1}(t)
$$

where $\epsilon$ is a constant depending on the fluorescence excitation rate and detection efficiency. The approximation is valid as long as $\epsilon t \ll 1$, which is true for the measurements reported below. Thus, at sufficiently low excitation intensity and for sufficiently short times $t$, there is no difference in temporal behavior between the interphoton time distribution and the fluorescence autocorrelation function.

In a rigorous sense, the derived solution for $s_{1}(t)$ and $g(t)$ is valid only for a constant value of the rate $k_{\text {eff }}$ and the coefficient $\epsilon$. However, in the experimental setup used, one has large variations in excitation intensity and detection efficiency over the detection region, so that the measured interphoton time distribution, $g(t)$, is proportional to a complicated average over all different positions of a molecule in the detection region:

$$
g(t) \propto \text { const }+\sum_{j=1}^{3} \tilde{\mathrm{c}}_{j}\left\langle\exp \left(-\lambda_{j} t\right)\right\rangle
$$

The angular bracket denotes averaging over all possible positions of the molecule within the detection volume, whereas this averaging and the constants $\tilde{c}_{j}$ are defined in such a way that the normalization $\langle 1\rangle=1$ holds. This notation is very convenient for rewriting the average of the exponential as an exponent of a cumulant expansion of averages:

$$
\left\langle\exp \left(-\lambda_{j} t\right)\right\rangle=\exp \left[-\left\langle\lambda_{j}\right\rangle t-\left\langle\left(\lambda_{j}-\left\langle\lambda_{j}\right\rangle\right)^{2}\right\rangle t^{2}-\ldots\right]
$$

If the distribution of values of $\lambda_{j}$ is sufficiently narrow so that $\left\langle\left(\lambda_{j}-\left\langle\lambda_{j}\right\rangle\right)^{2}\right\rangle \ll\left\langle\lambda_{j}\right\rangle^{2}$, the averaged exponent can be
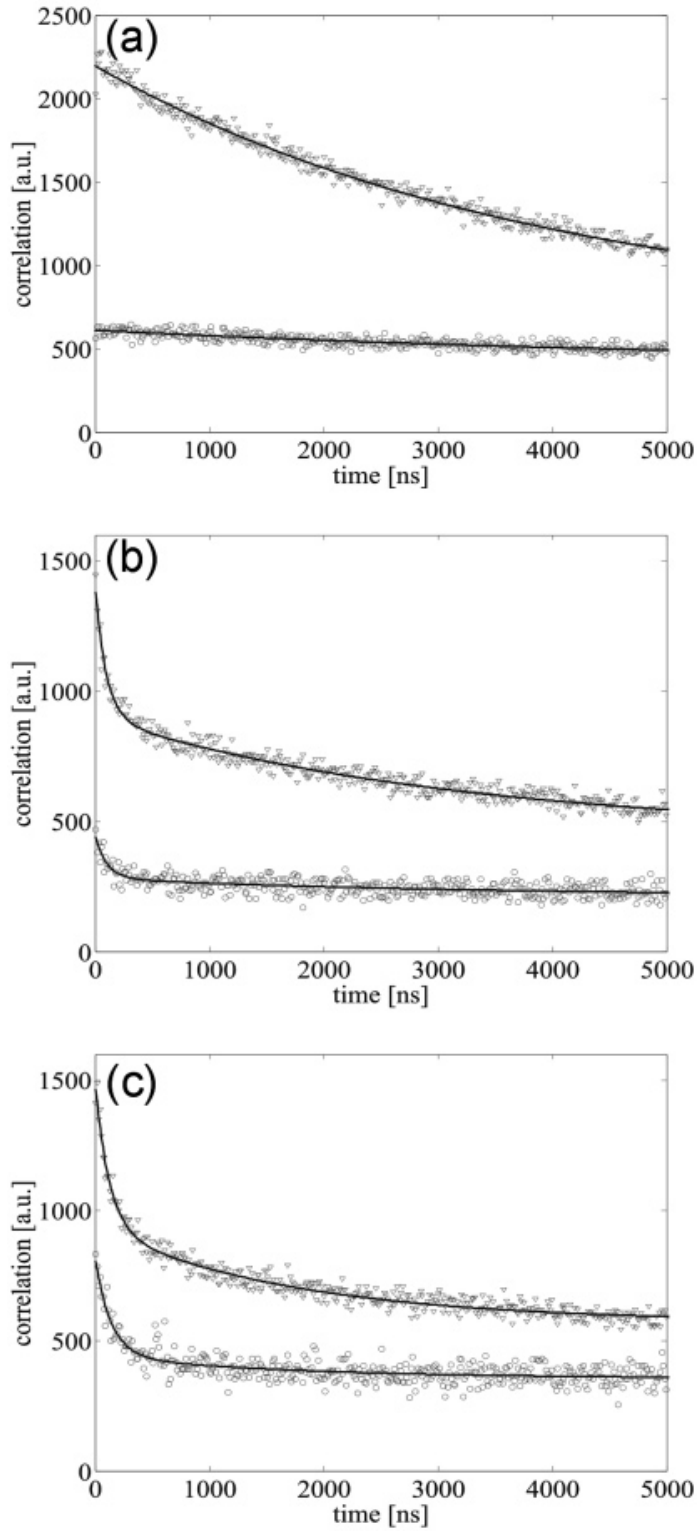

Figure 5. PDDs measured in PBS, $\mathrm{pH} 7.4$, at excitation intensities of 400 $\mu \mathrm{W}$ and $4 \mathrm{~mW}$ : (a) MR121-W9.F9; similar curves were obtained for MR121-W8·F8, and the pure dye MR121; (b) MR121-W9; (c) MR121W8. The lower curves refer to the low-intensity excitation measurement (circles) and the upper curves to the high-intensity excitation measurements (triangles).

approximated by the exponent of the average, and one observes a simple exponential decay behavior for $g(t)$.

Contact Formation Rates in Peptides. PDDs were recorded for $10^{-10} \mathrm{M}$ solutions of the four different polypeptides MR121-W8, MR121-W9, MR121-W8·F8, and MR121W9·F9 (Table 1), and the pure dye MR121 in PBS, pH 7.4 (Figure 5). Each measurement was performed at two different excitation intensities $(400 \mu \mathrm{W}$ and $4 \mathrm{~mW})$. For the highest excitation intensity used, the detected average photon count rate was below $10 \mathrm{kHz}$. For an estimated fluorescence detection efficiency of better than $10 \%$, and an absolute fluorescence quantum yield of larger than 0.1 , the average excitation rate is thus still by more than 3 orders of magnitude below optical saturation ( $\tau \approx 2 \mathrm{~ns}$ ). Hence, a linear relationship between excitation intensity and excitation rate was assumed. In all cases, the obtained PDDs, $g(t)$, could be perfectly fitted by either a 
Table 2. Fit Results for the Characteristic Times of the Applied Model Scheme Shown in Figure 4

\begin{tabular}{lllll}
\hline & $\begin{array}{c}1 / k_{\mathrm{t}} \\
(\mu \mathrm{s})\end{array}$ & $\begin{array}{l}1 / k_{\mathrm{t}}^{*} \\
(\mu \mathrm{s})\end{array}$ & $\begin{array}{l}1 / k_{+} \\
(\mathrm{ns})\end{array}$ & $\begin{array}{l}1 / k_{-} \\
(\mathrm{ns})\end{array}$ \\
\hline MR121 & & & & \\
MR121-W9·F9 & 5.80 & & & \\
MR121-W8·F8 & 5.80 & 3.18 & 120 & 267 \\
MR121-W9 & 5.80 & 1.74 & 152 & 742 \\
MR121-W8 & & & & \\
\hline
\end{tabular}

single exponential and a constant or a sum of three exponentials and a constant. This justifies the replacement of the average of the exponential by the exponential of the average in eq 1 , because otherwise significant deviations of measured PDDs from simple exponential decays would be expected. It shows also that, within the time window of $5 \mu \mathrm{s}$, the impact of translational diffusion on the autocorrelation $g(t)$ is still negligible. In addition, no polarization effects play any discernible role; i.e., the characteristic time of the rotational diffusion of a dye such as MR121 is faster in aqueous surroundings ( $<1 \mathrm{~ns})$ than the temporal resolution of the measurement of $1.2 \mathrm{~ns}$. First, the measurements of the pure dye and the peptides without Trp residues (MR121-W8·F8 and MR121-W9·F9) were analyzed. Figure 5a shows the PDDs obtained from the polypeptide conjugate MR121-W9·F9. Similar curves were obtained for MR121-W8·F8 and pure MR121. In the absence of fluorescence quenching by Trp residues, the PDDs were perfectly fitted by a monoexponential decay. The decay constant is solely determined by the triplet-state dynamics of the dye MR121 and is given by $\lambda_{1}=k_{\mathrm{t}}+k_{\text {eff. }}$. Because $k_{\text {eff }}$ is directly proportional to the excitation intensity, the measurement of the PDD at two different intensities with known ratio allows one to extract the rate constant $k_{\mathrm{t}}$ of the transition from the triplet $\left(\mathrm{T}_{1}\right)$ to the ground $\left(\mathrm{S}_{0}\right)$ state from the two fitted values of $k_{\mathrm{t}}+k_{\text {eff. }}$. Analysis of the data demonstrates that the triplet lifetime of MR121 is not influenced by the covalent attachment to a peptide. The pure MR121 and the two peptide conjugates without a Trp residue (MR121-W8·F8 and MR121-W9·F9) exhibit the same triplet lifetime of $5.8 \mu$ s (Table 2). In contrast, contact formation with the Trp residue in peptides MR121-W8 and MR121-W9 induces additional fluorescence dynamics. The measured PDDs for the two Trp-containing peptides (Figure 5b,c) were fitted with the full model as described by eq 1 , resulting in a threeexponential decay. With the assumption that the ground-state complexes formed between MR121 and Trp are essentially nonfluorescent, the ratio $K_{\text {eq }}=k_{+} / k_{-}$for the quenched peptides is given by the relative fluorescence quantum yields of the conjugates (Table 1). Furthermore, it is justified to assume that the triplet-state lifetime, $k_{\mathrm{t}}^{-1}$, of the dye MR121 is similar to the lifetime determined for the non-tryptophan-containing peptides, i.e., remains unchanged at $5.8 \mu$ s. Taking into account that the excitation intensities and thus the values of $k_{\text {eff }}$ differ by a factor of 10 in the two measurements for the same peptide, the remaining two fit parameters, $k_{\mathrm{t}}{ }^{*}$ and $k_{+}$, determine the characteristic roots, $\lambda_{j}$, via eq 1 . The resulting fit parameters $k_{\mathrm{t}}$, $k_{\mathrm{t}}{ }^{*}, k_{+}$, and $k_{-}$are summarized in Table 2 . The obtained association rate constants $k_{+}$of $1 / 120$ and $1 / 152 \mathrm{~ns}^{-1}$ for the peptides MR121-W9 and MR121-W8, composed of 15 and 20 amino acids, respectively, are of the same order of magnitude as those measured for end-to-end contact formation in peptides Cys-(Ala-Gly-Gln) ${ }_{n}$-Trp $(n=1-6) .{ }^{11}$ The dissociation rate constants $k_{-}$of $1 / 267 \mathrm{~ns}^{-1}$ for MR121-W9 and $1 / 742 \mathrm{~ns}^{-1}$ for MR121-W8 reflect the different quenching efficiencies of the Trp residues in positions 8 and 9, respectively. Furthermore, these values demonstrate that the association between the endlabeled dye and the Trp in position 8 is favored. Whereas the measured dissociation rate constants are influenced by the hydrophobic association of MR121 and Trp, it has to be discussed whether the experimental measured contact formation rate constants report on the rate constants of hydrophobic association rather than on the pertinent biopolymer chain mobility. First, the measured contact formation rate constants are similar to those reported for other peptides using ensemble time-resolved pump-probe absorption or fluorescence measurements. ${ }^{9-13}$ Second, it is unlikely that hydrophobic interactions across eight or nine amino acids impose contact formation between the dye and the Trp residue. As soon as contact formation occurs (initiated by backbone fluctuations in the peptide), hydrophobic interactions certainly stabilize the complex. This is directly reflected in the slower dissociation rate constants.

\section{Conclusions}

Our results and analysis show that measurements of the photon distance distributions of fluorescently labeled peptides containing a single Trp residue can accurately determine the rate constants of contact formation and dissociation with nanosecond temporal resolution. The advantages of the presently introduced fluorescence quenching based method comprise the ease of experimental monitoring in water under air with singlemolecule sensitivity. Because Trp forms ground-state complexes with the oxazine dye used and quenches within these complexes at a rate that is much faster than that of any other amino acid, fluorescence fluctuation analysis directly reveals the association and dissociation rate constants. We have assumed that quenching in the peptides via photoinduced electron transfer occurs only at van der Waals contact within the complexes. In future studies it will be important to determine how the measured contact formation rate constants depend on the peptide length and sequence and solvent conditions.

Acknowledgment. We thank Prof. K. H. Drexhage (Universität-Gesamthochschule Siegen) for providing the oxazine derivative MR121. This work was supported by the Bundesministerium für Bildung, Wissenschaft, Forschung und Technologie, BMBF (Grant 311864).

JA034040P 